\title{
Kinetics and Mass Spectrometric Measurements of Myoglobin Unfolding in Aqueous Ionic Liquid Solutions
}

\author{
Miranda C. Miller, Sylvia L. Hanna, Kelsey G. DeFrates, Olivia C. Fiebig, and Timothy D. Vaden* \\ Department of Chemistry and Biochemistry, Rowan University, 201 Mullica Hill Road, Glassboro, NJ, \\ 08028
}

*Corresponding Author: vadent@ rowan.edu

\begin{abstract}
Recent studies have characterized the effects of aqueous ionic liquids on myoglobin unfolding for the broader purposes of understanding their effects on protein structures, stabilities, and ultimately biocompatibilities for future applications. Here, we investigated the effects of four different ionic liquids (ILs) on the thermal stability, unfolding kinetics, and tertiary shape of myoglobin. We compared results for four different ILs: 1-butyl-3-methyl imidazolium tetrafluoroborate $\left(\mathrm{BMIBF}_{4}\right)$; 1-butyl-3-methyl pyrrolidinium tetrafluoroborate $\left(\mathrm{PyrrBF}_{4}\right) ;$ 1-ethyl-3-methyl imidazolium acetate (EMIAc); and tetramethylguanidinium acetate (TMGAc). Results showed that ILs accelerate myoglobin unfolding kinetics both through aqueous solution ionic strength effects and ionic liquid-specific effects. Arrhenius plots of observed rate constants reveal that some ILs lower the energy barrier to unfolding, possibly by destabilizing the native protein state. The magnitude of these ionic liquid effects correlates with their effects on protein thermodynamic stabilities. Hydrogen-deuterium exchange (HDX) experiments using ESI-MS showed that myoglobin exhibits a more open, and presumably less stable, tertiary shape in aqueous IL solutions. Overall, $\mathrm{BMIBF}_{4}$ and TMGAc exhibit the strongest effect on the myoglobin stability, unfolding rate, and tertiary structure while $\mathrm{PyrrBF}_{4}$ and EMIAc have weaker effects under our experimental conditions.
\end{abstract}

Keywords: Myoglobin; Ionic liquids; Protein unfolding 


\section{Introduction}

The structures and stabilities of proteins in aqueous ionic liquid (IL) solutions have been the focus of numerous recent reports in the literature [1-15]. Such studies are motivated by the utilization of ILs in several different biomedical and biochemical techniques [5, 16-21], where ILs are present as solutes in aqueous solution. ILs can participate in enzyme reactions, and also can be used to inhibit, activate, or otherwise modulate enzyme kinetics [3, 4, 22-26]. They have been used as potent antimicrobial drugs and drug delivery systems [17], and can affect protein aggregation [1, 5, 15, 20], including amyloid fibril formation [6, 12]. They have also been used for controlling protein conformational transitions [27-29] and assisting in the preparation of cellulose and other biomass for dissolution and processing [19, 30-32].

Significant data exists to enable design of ILs for many non-biochemical applications [33], but researchers are just beginning to compile comprehensive IL/protein data for prediction and design of biochemical/biomedical applications. A number of groups have studied different ILs series and their effects on protein stability $[11,16,34-36]$, IL-protein surface interactions $[9,26,37,38]$, and protein thermodynamic stability in aqueous IL solutions [5, 7]. Low-concentration ILs in aqueous solution often do not denature proteins, but can destabilize their structures as demonstrated by numerous thermodynamic studies $[5,7,8,10,15,39]$. Several recent reports have focused on IL-protein interactions in $\alpha$-helical proteins and their role in protein destabilization $[2,3,8,11,40]$. In this context myoglobin, an $\alpha$-helical protein with a heme moiety, has received recent attention in both applied and fundamental studies [7, 11, 27, 39, 41-43]. Protein stabilization and/or destabilization by ILs in aqueous solution is often explained with the Hoffmeister anion series [44], but myoglobin may not exactly follow this series $[35,36,39]$. The effects of different ILs, especially new biocompatible ILs, on myoglobin's tertiary structure and dynamics are not yet completely understood and additional experiments can provide further insights.

While many reports have characterized the thermodynamic stabilities of proteins in aqueous IL solutions, relatively few have studied IL effects on protein unfolding kinetics. Myoglobin unfolding in high concentrations of urea denaturant proceeds slow enough to enable relatively facile kinetics 
measurements [45, 46]. Such kinetics experiments have elucidated how hydrophobic and aromatic interactions with myoglobin residues near the heme group can destabilize the protein and increase unfolding rate constants [45, 46]. Kinetics measurements in the presence of aqueous ILs can thus offer insight into different IL effects on myoglobin, especially as hydrophobic/aromatic interactions have been identified as contributors to protein destabilization [8, 24, 36, 39, 47]. Ultimately, kinetics measurements can help elucidate IL effects on protein folding pathways and point to mechanisms of kinetic stabilization and protein/IL-selective unfolding.

Insights into protein-IL interactions and their effects on tertiary structures can also be obtained with mass spectrometry and hydrogen-deuterium exchange (HDX) experiments. In an HDX experiment, a protein in $\mathrm{D}_{2} \mathrm{O}$ (heavy water) exchanges its $\mathrm{H}$-atoms (typically from $\mathrm{N}-\mathrm{H}$ groups) for D-atoms. Structural information is obtained due to exposed "surface" N-H groups exchanging faster than buried residues, and hence the number of D-atoms added to a protein during a given reaction time elucidates the structure as tightly folded (few D-atoms added), slightly loosened (more D-atoms added), or completely opened/unfolded (many D-atoms added) [48-50]. Nuclear magnetic resonance (NMR)-based HDX measurements have been used to characterize IL interactions with a short peptide [8], but for proteins mass spectrometry-based HDX measurements are more simple and can provide direct qualitative information regarding the extent of tertiary-level protein structural changes in aqueous IL solutions.

In this work, we investigate myoglobin in aqueous IL solutions using unfolding kinetics measurements and HDX experiments. We compare results for four different ILs: 1-butyl-3-methyl imidazolium tetrafluoroborate $\left(\mathrm{BMIBF}_{4}\right) ;$ 1-butyl-3-methyl pyrrolidinium tetrafluoroborate $\left(\mathrm{PyrrBF}_{4}\right)$; 1-ethyl-3methyl imidazolium acetate (EMIAc); and tetramethylguanidinium acetate (TMGAc). In a previous report [7], we quantified myoglobin thermodynamic stabilities in aqueous solutions of $\mathrm{BMIBF}_{4}$ and EMIAc and showed that $\mathrm{BMIBF}_{4}$ destabilizes myoglobin while EMIAc has minimal effect on the protein stability. The ILs are chosen to offer a broad array of ILs and to compare different cations and anions. $\mathrm{BMIBF}_{4}$ and PyrrBF 4 contain the same anion $\left(\mathrm{BF}_{4}^{-}\right)$and differ only in the aromaticity of the cation (imidazolium is aromatic while pyrrolidinium is not). EMIAc and TMGAc both contain the acetate anion ( $\left.\mathrm{Ac}^{-}\right)$and differ 
in the cation. Notably, TMGAc has been designed as a biocompatible IL for protein purification [51] but has not previously been used with myoglobin.

The combination of routine protein stability studies in aqueous IL solutions with unfolding kinetics and HDX measurements offers broad insights into how destabilization by ILs affects protein shapes and unfolding transitions. In this work we present this combined analysis for the myoglobin protein. Unfolding kinetics measurements demonstrate how different ILs affect unfolding rates and unfolding energy barriers, and HDX measurements quantify the extent to which different ILs affect protein shapes.

\section{Experimental Methods}

\subsection{Sample Preparation}

Horse-skeletal myoglobin was obtained from Sigma-Aldrich and used without further purification. $\mathrm{BMIBF}_{4}, \mathrm{EMIAc}$, and $\mathrm{PyrrBF}_{4}$ were obtained from Sigma-Aldrich ( $\geq 98 \%$ purity) and were also used without purification. TMGAc was synthesized using a reported procedure [51]. Tetra-methyl-guanidine (Alfa Aesar) and glacial acetic acid (Fisher Scientific) were mixed in a 1:1 molar ratio in $50 \mathrm{~mL}$ ethanol and stirred at room temperature overnight. The ethanol was removed by rotary evaporation at $40{ }^{\circ} \mathrm{C}$. The identity and purity ( $\geq 95 \%$ ) of the TMGAc was verified by NMR spectroscopy.

For all protein studies, solutions were prepared containing constant myoglobin concentrations $(0.2$ $\mathrm{mg} / \mathrm{mL}$ ) in $20 \mathrm{mM}$ pH-7 phosphate buffer. ILs were added from stock solutions of the ILs in aqueous solution and $20 \mathrm{mM} \mathrm{pH-7}$ phosphate buffer. IL stock solutions were prepared fresh and used immediately prior to experiments to prevent effects of IL decomposition (e.g., hydrolysis of $\mathrm{BMIBF}_{4}$ to generate HF). $\mathrm{pH}$ measurements showed that the ILs in aqueous solution had minimal effects on solution $\mathrm{pH}$ beyond expected ionic strength effects: the $\mathrm{pH}$ decreased to $~ 6.8-6.9$ in the aqueous IL solutions.

\subsection{Temperature Stability Studies}

Preliminary thermodynamic stability studies were performed in a temperature-controlled Jasco J-810 circular dichroism (CD) spectrometer. For each sample, the absorbance and CD spectra were simultaneously measured from $300-500 \mathrm{~nm}$ at different temperatures from 25 to $93{ }^{\circ} \mathrm{C}$. The sample was 
placed in a sealed cuvette to prevent water evaporation. The heme moiety in myoglobin exhibits a strong absorbance band at $409 \mathrm{~nm}$ that also displays a strong positive CD signal [52]. While both the $409 \mathrm{~nm}$ absorbance and CD intensity can be used to quantify the fraction of the folded protein [52-54], we used the absorbance values for consistency throughout this work. The fraction of folded protein was computed from $409 \mathrm{~nm}$ absorbance following previous reports [7, 54]. Briefly, the 409 absorbance in the spectrum of native myoglobin in buffer (shown in Figure S1) is assumed to correspond to $100 \%$ folded protein and the 409 absorbance in the spectrum of myoglobin with 8.0 M urea (also shown in Figure S1) is assumed to correspond to $0 \%$ folded protein. The folded fraction was computed as

$$
\text { Fraction Folded }=\frac{A_{409}-A_{u}}{A_{f}-A_{u}}
$$

This method, in which $\mathrm{A}_{409}$ is the absorbance, $\mathrm{A}_{\mathrm{u}}$ is the $409 \mathrm{~nm}$ absorbance of the unfolded protein, and $\mathrm{A}_{\mathrm{f}}$ is the absorbance of the folded protein, corrects for the residual $409 \mathrm{~nm}$ absorbance of the unfolded protein and was used for all fraction folded calculations.

For reference, myoglobin stability studies were also performed using urea titrations in the presence of some ILs. For each experiment, $\sim 10$ solutions were prepared with increasing urea concentrations from 0 to $9.0 \mathrm{M}$, and for each experiment the solution contained a constant $150 \mathrm{mM}$ concentration of aqueous IL. Urea (VWR) was added from a $10 \mathrm{M}$ stock solution in $20 \mathrm{mM} \mathrm{pH}-7$ phosphate buffer. As myoglobin unfolding in urea is slower than in $\mathrm{GuHCl}$ (vide infra), samples were incubated for two hours prior to measurement. Absorbance spectra were measured with a Perkin-Elmer Lambda-35 spectrometer with a solution of corresponding aqueous IL as blank.

\section{$\underline{2.3 \text { Unfolding Kinetics Measurements }}$}

To measure unfolding kinetics, myoglobin samples were prepared in quartz cuvettes in aqueous IL solutions without urea. Appropriate aliquots of urea from the $10 \mathrm{M}$ stock solution were then added and the absorbance at $409 \mathrm{~nm}$ (proportional to the folded protein concentration [53, 54]) was measured as a function of time after urea addition with continuous stirring. Reported urea and IL concentrations are the final values after urea addition. These experiments were performed using Vernier SpectroVis Plus 
spectrometers placed on magnetic stirring plates. IL concentrations were varied from $0-400 \mathrm{mM}$ and data sets were acquired with both 6 and $8 \mathrm{M}$ urea. Because ionic strength is known to affect unfolding kinetics, $\mathrm{NaCl}$ control experiments were performed with the same concentrations $(0-400 \mathrm{mM})$ [55].

Temperature-dependent kinetics were measured in the temperature-controlled Jasco J-810 CD spectrometer. Both the UV absorbance and CD signal at $409 \mathrm{~nm}$ were monitored versus time after urea addition at different temperatures with continuous stirring. Unfolding kinetics curves were generated based on the 409 absorbance.

\subsection{HDX Experiments}

Mass spectrometry experiments were performed using an Agilent 1100 Series LC/MSD trap with a needle voltage of $3.5 \mathrm{kV}$ and a continuous LC flow rate of $100 \mu \mathrm{L} / \mathrm{min}$. Myoglobin samples were prepared at $1 \mathrm{mg} / \mathrm{mL}$ concentration in $20 \mathrm{mM} \mathrm{pH}-7$ phosphate buffer in the presence of different concentrations of aqueous ILs. Proteins under various conditions, with or without ILs in buffer solution, can be rapidly separated from the other solution components (ILs, phosphate buffer salts, and denaturants) with size-exclusion or desalting LC columns [50]. To accomplish this, samples ( $8 \mu \mathrm{L}$ aliquots) were injected and passed through an Acclaim PolarAdvantage II desalting column to remove buffer and IL salts. Initially, the mobile phase was water with $0.1 \%$ formic acid to remove the salts, which were discarded into waste. The mobile phase was then switched to acetonitrile with $0.1 \%$ formic acid to elute the protein, which was directed into the ESI-MS instrument. The $\mathrm{pH}$ of the injected myoglobin solution after mixing with the $0.1 \%$ formic acid mobile phase was about 2.5 , which was low enough to quench the H/D exchange immediately after injection.

Hydrogen-deuterium exchange (HDX) experiments were performed by adding $0.9 \mathrm{~mL}$ of $\mathrm{D}_{2} \mathrm{O}$ (Cambridge Isotope Laboratories, $99.9 \%$ ) to $0.1 \mathrm{~mL}$ of the myoglobin samples prepared above $\left(90 \% \mathrm{D}_{2} \mathrm{O}\right.$ present for all experiments). Samples were stirred vigorously for a fixed time length ( 10-40 seconds) and quenched by adding buffer to lower the $\mathrm{pH}$ to less than 2.5. Samples were then injected immediately and 
analyzed with the LC/ESI-MS system. One measurement was performed immediately prior to mixing with $\mathrm{D}_{2} \mathrm{O}$ for $\mathrm{t}=0$ data points.

\section{Results and Discussion}

\subsection{Myoglobin Stability in Different Aqueous Ionic Liquid Solutions}

We first discuss the effects of the aqueous ILs on the stability of myoglobin. These results supplement other thermal stability studies in the literature and help contextualize the kinetics and HDX experiments. We obtained protein thermal denaturing curves for myoglobin in different aqueous ionic liquid solutions by monitoring $409 \mathrm{~nm}$ absorbance values at increasing temperatures as described in Section 2. The results are summarized in Figure 1. In the absence of ILs (black squares in Figs. 1A-1D), myoglobin unfolds at $\sim 80{ }^{\circ} \mathrm{C}$. The rest of the data in Figure 1 yield clear and direct insight into the destabilizing effects of the aqueous ILs.

At $25{ }^{\circ} \mathrm{C}$ in $\mathrm{pH}$-buffered solution, myoglobin remains folded in the presence of all the ILs at all concentrations up to $300 \mathrm{mM}$. This is consistent with previous reports $[8,16,28,39]$. The results for EMIAc (Figure 1A) show that this IL does not have a detectable effect on the unfolding temperature. This is consistent with our previous report [7] that indicates EMIAc has only a minor effect on the myoglobin stability. However, the addition of some ILs clearly lowers the unfolding temperature, indicating protein destabilization. The TMGAc (Figure 1B) and $\mathrm{PyrrBF}_{4}$ (Figure 1C) lower the unfolding temperature by $\sim 5-10{ }^{\circ} \mathrm{C}$ while the $\mathrm{BMIBF}_{4}$ (Figure 1D) lowers the unfolding temperature by $\sim 25^{\circ} \mathrm{C}$. Hence, EMIAc has negligible destabilizing effects on myoglobin, TMGAc and $\mathrm{PyrrBF}_{4}$ have minor destabilizing effects, and $\mathrm{BMIBF}_{4}$ has major destabilizing effects.

\subsection{Kinetics of Urea-Induced Unfolding in Aqueous Ionic Liquid Solutions}

We used urea-based unfolding experiments to study how the IL destabilization demonstrated in Figure 1 affects the myoglobin unfolding kinetics. Equilibrium urea-denaturing measurements, shown in Figure 2, provide information about how urea affects the protein unfolding equilibrium in different aqueous IL 
solutions. Results are shown only for the two extreme cases of EMIAc (little or no destabilization) and $\mathrm{BMIBF}_{4}$ (major destabilization).

The results obtained in the absence of ILs (triangles) show that myoglobin unfolds with $\sim 6.0 \mathrm{M}$ urea (inflection point of denaturing curve). The presence of ILs (at $0.15 \mathrm{M}$ concentration) causes the protein to unfold at lower urea concentrations in a manner consistent with the results of Figure 1. The unfolding midpoint is $\sim 5.5 \mathrm{M}$ urea with EMIAc (minor decrease from myoglobin without $\mathrm{IL}$ ) and $\sim 3.5 \mathrm{M}$ with $\mathrm{BMIBF}_{4}$ (major decrease from myoglobin without IL). Importantly, Figure 2 shows that in all cases myoglobin completely unfolds in 8.0 M urea. Time-based unfolding measurements in $8.0 \mathrm{M}$ urea can therefore provide direct information regarding the IL effects on kinetics.

The results of the unfolding kinetics measurements are summarized in Figure 3. For all kinetics experiments, we compute the fraction of folded protein from the $409 \mathrm{~nm}$ absorbance by assuming that the fraction is 1 before addition of urea and 0 after unfolding is complete (consistent with Figure 2) to correct for the residual $409 \mathrm{~nm}$ absorbance values of unfolded myoglobin. A data set with error bars and the exponential fit is included in the Supplementary Material as Figure S2. For all ILs, increasing the IL concentration results in increased unfolding rates.

The unfolding decay curves can be fit to a single exponential decay function with an observed rate constant $\mathrm{k}_{\mathrm{OBs}}$. Under the two-state model of protein folding $[8,56], \mathrm{k}_{\mathrm{OBS}}$ is the sum of $\mathrm{k}_{\mathrm{f}}$ and $\mathrm{k}_{\mathrm{u}}$, the folding and unfolding rate constants, respectively [57]:

$$
k_{\text {OBS }}=k_{f}+k_{u}
$$

In Equation (2), both $k_{f}$ and $k_{u}$ depend on the concentration of denaturant. The fundamental values of $k_{f}$ and $\mathrm{k}_{\mathrm{u}}$ in absence of denaturant can be measured by performing a chevron analysis, which requires measurements of $\mathrm{k}_{\mathrm{OBS}}$ as a function of denaturant concentration $[55,58]$. Due to limitations on protein and urea consumption, extensive concentration-dependent kinetics measurements were not performed; we only used 6.0 $\mathrm{M}$ and $8.0 \mathrm{M}$ urea and focus our analysis on the $8.0 \mathrm{M}$ data set. At $8.0 \mathrm{M}$ urea 
concentration, the $\mathrm{k}_{\mathrm{f}}$ term in Equation (2) is very small and the value of $\mathrm{k}_{\mathrm{OBS}}$ is dominated by the $\mathrm{k}_{\mathrm{u}}$ term (which clearly depends on the urea concentration) [59].

From Figure 3, myoglobin unfolding is clearly accelerated in aqueous IL solutions, but it is well-known that protein unfolding kinetics are affected by solution ionic strength [55]. Thus, the increase in $\mathrm{k}_{\mathrm{OBS}}$ values by the addition of electrolytic ILs into aqueous solution could in principle be due simply to increased ionic strength. Therefore we also performed urea-based unfolding experiments with $\mathrm{NaCl}$, in which the increase in $\mathrm{k}_{\mathrm{OBS}}$ is purely related to increased ionic strength.

The $\mathrm{k}_{\mathrm{OBS}}$ values derived from the experimental data in Figure 3, including the $\mathrm{NaCl}$-control values, are summarized in Figure 4. $\mathrm{k}_{\mathrm{OBS}}$ rate constants were obtained by fitting both the raw data (409 $\mathrm{nm}$ absorbance vs time) and the folded fraction data (shown in Figure 3). No differences in rate constants were observed between the two data sets. These rate constants were obtained by fitting the Figure 3 data to the single-exponential decay function of Fraction Folded versus time using a function-fitting algorithm in Origin 8.5; the exponential decay constant is $\mathrm{k}_{\mathrm{OBS}}$. An example of the exponential fit is shown in Figure S2.

The rate constants in Figure 4 increase with increasing IL concentration (Fig. 4A). The increase in $\mathrm{k}_{\mathrm{OBS}}$ from ionic strength effects alone should be proportional to $\sqrt{I}$ due to the electrostatic screening effect [55]. The unfolding data in the presence of $\mathrm{NaCl}$ and EMIAc appear to closely follow the $\sqrt{I}$ dependence. This is highlighted by plotting $\mathrm{k}_{\mathrm{OBS}}$ versus $\sqrt{I}$ in Figure $4 \mathrm{~B}$. The data for $\mathrm{NaCl}$ and EMIAc clearly follow a linear trend. This immediately suggests that the effect of EMIAc on myoglobin unfolding is of a predominantly ionic strength nature. With $\mathrm{PyrrBF}_{4}$ and $\mathrm{TMGAc}$, the $\mathrm{k}_{\mathrm{OBS}}$ values appear to deviate from the $\mathrm{NaCl}$ trend at higher IL concentration and are $\sim 15-20 \%$ higher than the values in $\mathrm{NaCl}$ at 300 and $400 \mathrm{mM}$. This suggests that the $\mathrm{PyrrBF}_{4}$ and TMGAc have some minor additional "IL" effect on the unfolding kinetics beyond the simple ionic strength effect. With $\mathrm{BMIBF}_{4}$, the $\mathrm{k}_{\mathrm{OBS}}$ values are significantly higher than the $\mathrm{NaCl}$-control values, especially at IL concentrations above $200 \mathrm{mM}$. The influence of $\mathrm{BMIBF}_{4}$ on the unfolding transition is clearly stronger than a simple ionic strength effect. 
From Figure 4, some ILs have a significant effect on myoglobin unfolding kinetics while others appear to only increase the solution ionic strength. To further investigate these effects we performed temperature-dependent kinetics experiments as described in Section 2. Arrhenius plots of the $\mathrm{k}_{\mathrm{OBS}}$ values measured from 25 to $55^{\circ} \mathrm{C}$ are shown in Figure 5. For clarity, only the data with no additive, $\mathrm{NaCl}$, and $\mathrm{BMIBF}_{4}$ are shown. The complete data set is included in the Supplementary Material as Figure S3. From Figure 5, it is clear that the data with and without $\mathrm{NaCl}$ (red circles versus blue triangles) have different intercepts but essentially the same slopes. However, the data with $\mathrm{BMIBF}_{4}$ (black squares) has a different slope from the other two data sets. Note that all the data sets in Figure S3 have different intercepts from the buffer-only data.

The slope of the Arrhenius plots in Figure 5 is not exactly related to the unfolding transition activation energy because $\mathrm{k}_{\mathrm{OBS}}$ is not exactly the fundamental unfolding rate constant (i.e., $\mathrm{k}_{\mathrm{u}}$ in absence of urea). Nevertheless, as $\mathrm{k}_{\mathrm{OBS}}$ is dominated by the $\mathrm{k}_{\mathrm{u}}$ value at $8.0 \mathrm{M}$ urea, it is appropriate to discuss the slopes in terms of energy barriers to unfolding. The plots in Figure 5 were fit with a linear equation using Origin 8.5. The linear slope is $-\mathrm{E}_{\mathrm{A}} / \mathrm{R}$, where $\mathrm{E}_{\mathrm{A}}$ is the activation energy, or energy barrier. The energy barrier values derived from the Arrhenius plots in Figs. 5 and S3 are summarized in Table 1. The errors are computed from the errors in the Arrhenius plot fits. It appears that some ILs have negligible impact on the unfolding energy barrier while other ILs significantly lower the barrier. While the errors are rather large, it is clear that the $\mathrm{BMIBF}_{4}$ has the most dramatic energy barrier-lowering effect.

Table 1. Unfolding energy barriers $(\mathrm{kJ} / \mathrm{mol})$ derived from the linear fits in Figure 5 and Figure S3.

\begin{tabular}{|c|c|c|}
\hline $\mathbf{2 0 0} \mathbf{~ m M} \mathbf{~ I L}$ & $\left.\mathbf{E}_{\mathbf{A}} \mathbf{( k J / m o l}\right)$ & Error $(\mathbf{k J} / \mathbf{m o l})$ \\
\hline No IL & 115 & \pm 14 \\
\hline NaCl & 103 & \pm 14 \\
\hline TMGAc $^{\text {BMIBF }}$ & 78 & \pm 14 \\
\hline PyrrBF $_{4}$ & 71 & \pm 13 \\
\hline EMIAc $^{2}$ & 91 & \pm 14 \\
\hline
\end{tabular}


A previous study has shown that sodium dodecyl sulfate (SDS) has a strong protein destabilizing effect and accelerates protein unfolding kinetics and lowers unfolding activation energies by $\sim 40 \mathrm{~kJ} / \mathrm{mol}$ at $\sim 100$ $\mathrm{mM}$ [55]. In this context the IL effects on myoglobin unfolding appear relatively weaker than surfactant effects. However, it does appear that there is a correlation between the destabilization effects of the different aqueous ILs (Figure 1) and the lowered unfolding energy barriers. $200 \mathrm{mM}$ EMIAc and NaCl, which have no effects on the stability, lower the energy barrier by less than $20 \mathrm{~kJ} / \mathrm{mol}$, while $200 \mathrm{mM}$ $\mathrm{BMIBF}_{4}$, which clearly has the strongest destabilization effect, lowers the energy barrier by $\sim 40 \mathrm{~kJ} / \mathrm{mol}$.

\subsection{HDX-MS Results for Myoglobin in Ionic Liquid Solutions}

The ILs in aqueous solution do not denature myoglobin but can clearly destabilize the protein structure and lower the energy barrier to unfolding. These results are consistent with previous reports showing that protein-IL interactions result in more open protein structures $[1,2,8,39,60,61]$ and suggest that the myoglobin structure is itself weakened by the IL molecules in solution. We investigated the extent of the protein structural changes induced by ILs using MS-based HDX experiments.

Briefly, the pH-buffered myoglobin-IL solution was mixed with an aliquot of $\mathrm{D}_{2} \mathrm{O}$ and the resultant mass changes were measured as a function of time. If the protein tertiary shape changes, a greater fraction of easily-exchangeable hydrogen atoms are exposed to $\mathrm{D}_{2} \mathrm{O}$ in solution allowing $\mathrm{H}-\mathrm{D}$ exchange and resulting in an increase in the total mass of the protein. For example data, Figure 6 shows the ESI-MS spectra of myoglobin (+16 charge state) before and after HDX in the absence (Fig. 6A) and presence (Fig. 6B) of $\mathrm{BMIBF}_{4}$. In both cases, the HDX results in an increase in mass of $\sim 0.5-1.5 \mathrm{~m} / \mathrm{z}$ unit, which corresponds to the addition of $\sim 10-20$ D-atoms. Note that additional peaks at 1066 and $1075 \mathrm{~m} / \mathrm{z}$ in Fig. 6B can be attributed to myoglobin- $\mathrm{BF}_{4}$ adducts. Comparing Figs. $6 \mathrm{~A}$ and $6 \mathrm{~B}$, it appears that more Datoms are added to the protein in the presence of the IL (larger mass shift after $\mathrm{D}_{2} \mathrm{O}$ addition), which immediately suggests faster HDX exchange and further suggests that the protein tertiary structure is more open in the $\mathrm{BMIBF}_{4}$ solution. 
The results of the HDX measurements versus time are presented in Figure 7. We computed the average protein mass and standard deviation at each time-point by calculating the weighted-average mass of each charge state, multiplying this weighted-average mass by the protein charge, and then averaging together the different total protein mass values for the different charge states. To calculate the weighted-average mass for a given charge state, we defined a mass window of \pm 3 amu around the maximum (e.g., 1060.5 $\mathrm{m} / \mathrm{z}$ for the bottom trace in Fig. 6A) and within this window computed the average of the ion masses each weighted by their intensities. For each time point we used the same charge states from +9 to +22 .

Prior to the addition of $\mathrm{D}_{2} \mathrm{O}(\mathrm{t}=0)$, all samples exhibit protein masses within the measurement error of each other. After $\mathrm{D}_{2} \mathrm{O}$ addition, the protein masses increase within the first $\sim 10-20$ seconds and become roughly constant after this time, suggesting that the fast-exchanging amides react in this time period. Using the 30 -seconds time point as an approximation of equilibrium exchange, the mass increases by $\sim 15$ amu with no IL in solution, corresponding to the addition of $\sim 15 \mathrm{D}$-atoms. With ILs present, the protein mass increases more significantly: $\sim 25$ D-atoms are added to myoglobin in the presence of EMIAc and TMGAc (1.5-fold greater H/D exchange compared to myoglobin without ILs) and 35 D-atoms are added to myoglobin in the presence of $\mathrm{BMIBF}_{4}$ (more than 2-fold greater H/D exchange compared to myoglobin without ILs). It appears that the HDX addition to myoglobin in the presence of $\mathrm{PyrrBF}_{4}$ is similar to myoglobin without ILs. While the error bars are large (due to low MS resolution), the differences between these data sets are significant.

The HDX-based mass increases in Figure 7 show that the amides exchange D-atoms faster and to a greater extent in the presence of ILs. This immediately suggests that the ILs in aqueous solution cause some structural change in myoglobin, resulting in looser, more open protein tertiary structures with more exposed peptide backbones. We performed control experiments with $200 \mathrm{mM} \mathrm{NaCl}$, shown in Figure S4 in the Supplementary Material. The mass increase with $\mathrm{NaCl}$ is the same as without any IL, indicating that the greater H/D exchange is not due to increased ionic strength and that the data in Figure 7 indeed illustrate the effects of the different ILs on the protein structure. The degree of myoglobin structural "loosening" is strongest with $\mathrm{BMIBF}_{4}$ and TMGAc and less pronounced with EMIAc and PyrrBF 4 , which 
is clearly consistent with the previous thermodynamic (Figure 1) and kinetics (Figs. 3-5) results. Notably, the "protein-loosening" effect is almost as strong with TMGAc as it is with $\mathrm{BMIBF}_{4}$, which is consistent with the results in Table 1 that show that TMGAc lowers the energy barrier to unfolding almost as much as $\mathrm{BMIBF}_{4}$.

\section{$\underline{3.4 \text { Discussion }}$}

The thermal stability analysis presented in Figure 1 offers quick insight into how the different ILs affect myoglobin. $\mathrm{BMIBF}_{4}$ destabilizes myoglobin while EMIAc has minimal effect. Studies have shown that the aromatic imidazolium cations can bind to helical proteins, and the alkyl chains destabilize the protein secondary/tertiary structures $[26,40,47]$. Thus it makes sense that $\mathrm{BMI}^{+}$, with the longer butyl chain, destabilizes the protein more than $\mathrm{EMI}^{+}$with its ethyl chain. Regarding the anion, $\mathrm{Ac}^{-}$is in the middle of the Hoffmeister series $[39,44]$ and $\mathrm{BF}_{4}^{-}$can bind directly to the protein [37]. Again, it makes sense that the $\mathrm{Ac}^{-}$-containing IL has less destabilizing effect than the $\mathrm{BF}^{-}$-containing IL.

The comparison in Figure 1 with the newly-studied ILs PyrrBF 4 and TMGAc offers further insight. $\mathrm{PyrrBF}_{4}$ has a significantly reduced destabilization effect relative to $\mathrm{BMIBF}_{4}$, immediately indicating that the aromaticity of the $\mathrm{BMI}^{+}$cation is very influential in destabilizing myoglobin (likely through aromatic interactions with the protein). TMGAc has a somewhat stronger destabilization effect relative to EMIAc (though its effect is weaker than $\mathrm{BMIBF}_{4}$ ), illustrating the importance of the $\mathrm{TMG}^{+}$cation. $\mathrm{TMGAc}$ may affect the water hydrogen bond network [51] and may destabilize the protein structure in a similar manner to the well-known denaturant guanidinium hydrochloride $[62,63]$.

The nature of ILs in aqueous solution and their effects on proteins is of fundamental interest, and the kinetics study provides data and insights. ILs in water can dissociate as strong electrolytes, but may also be found as contact ion pairs or ion-cluster aggregates [64-66]. As aqueous electrolytes, ILs could have effects simply related to increased solution ionic strength. By comparing myoglobin unfolding kinetics in aqueous IL solutions with kinetics in aqueous $\mathrm{NaCl}$ solutions, it is clear that some ILs affect myoglobin primarily through increased ionic strength while other ILs have some extra IL-specific effect. This conclusion is supported by the observed unfolding rate constant, $\mathrm{k}_{\mathrm{OBS}}$, and its $\mathrm{IL}$ (or $\mathrm{NaCl}$ ) concentration 
dependence (Figure 4), and by the IL-specific energy barrier-lowering effects shown in Table 1. It is clear that $\mathrm{BMIBF}_{4}$ and, to a lesser extent, TMGAc, lower the energy barrier to protein unfolding. This is consistent with the energy destabilization reported previously and shown in Figure 1. Decreased folded protein stability will result in a decreased barrier for unfolding even if the mechanism and transition state (and any intermediates, etc) are unaffected.

The results presented here and elsewhere consistently show that myoglobin remains folded in aqueous solutions of ILs but may be thermodynamically and kinetically destabilized [7, 11, 39]. The nature and extent of the native structure destabilization is elucidated by the HDX measurements. In aqueous IL solutions, myoglobin exhibits greater H/D exchange, and the degree of extra H/D exchange correlates with the IL-based decreases in thermodynamic stability (Figure 1) and energy barrier-lowering IL effects (Table 1). Based on the average protein mass measurements in Figure 7, we conclude that myoglobin adopts a more open structure in aqueous IL solutions. Importantly, the extent to which the myoglobin structure is "loosened" (based on the extent of H/D exchange) correlates with the extent to which the protein destabilizes the protein structure and lowers the energy barrier to unfolding $\left(\mathrm{BMIBF}_{4}>\mathrm{TMGAc}_{\mathrm{T}}>\mathrm{PyrrBF}_{4}>\mathrm{EMIAc}\right)$.

\section{Conclusions}

The effects of ILs on proteins in aqueous solution strongly depend on the specific IL molecular cation and anion. Recent studies investigating proteins in IL environments have focused on myoglobin to elucidate general and specific IL influences on $\alpha$-helical protein characteristics. We have used thermal denaturing studies, urea-based unfolding kinetics measurements, and HDX experiments to investigate the effects of four different ILs on myoglobin in aqueous solution. The ILs have been chosen to compare destabilizing with non-destabilizing systems $\left(\mathrm{BMIBF}_{4}\right.$ vs EMIAc) and also compare ILs containing the same anion but different cations $\left(\mathrm{BMIBF}_{4}\right.$ vs $\mathrm{PyrrBF}_{4}$ and EMIAc vs TMGAc). Thermal denaturing studies show that the $\mathrm{BMI}^{+}$cation has a strong destabilizing effect on myoglobin and, based on the observation that $\mathrm{PyrrBF}_{4}$ does not stabilize myoglobin as strongly as $\mathrm{BMIBF}_{4}$, the aromaticity of the 
imidazolium cation is important. Also noteworthy is the observation that TMGAc, a new biocompatible IL, has some destabilizing effect on myoglobin.

The key conclusions from this work concern the nature and extent of the IL effects on myoglobin. Unfolding kinetics measurements show that ILs accelerate protein unfolding, which would be expected for any electrolytic species as increased solution ionic strength screens electrostatic fields and increases unfolding rates. We can separate IL-specific effects from ionic strength effects by comparing unfolding rate constants in aqueous IL solutions to those in $\mathrm{NaCl}$ control solutions. The ILs that do not significantly destabilize myoglobin (EMIAc) appear to increase unfolding rates only due to the ionic strength effects, while the ILs that destabilize myoglobin have strong additional IL-specific effects. These IL-specific effects include lowering the energy barrier to unfolding, as demonstrated by the Arrhenius plots. These IL-specific effects likely include both protein-IL interactions and hydration structure alterations.

The extent of the IL effects on myoglobin's structure was further characterized using the HDX experiments, which utilize H/D exchange with exposed surface amide groups. The ILs in aqueous solution appear to result in a more open, less stable myoglobin structure with more exposed surface amide groups. The degree to which the ILs cause more H/D exchange correlates with their destabilization effects and the magnitudes of the IL-specific kinetics effect. This work demonstrates how ILs affect myoglobin unfolding kinetics by destabilizing the native protein and lowering the energy barrier to unfolding.

\section{Acknowledgement}

The authors gratefully acknowledge Rowan University and NSF grant CHE-1362493 for funding this research project. The authors also thank Dr. Gregory Caputo for advice and comments on the manuscript and Mr. Kevin Pang for assistance with the kinetics measurements.

\section{References}

[1] T. Takekiyo, K. Fukudome, K. Yamakazi, H. Abe, Y. Yoshimura, Chem. Phys. Lett., 602 (2014) 22-27. [2] T. Takekiyo, A. Nihei, K. Yamazaki, M. Aono, H. Abe, Y. Yoshimura, J. Solution Chem., 43 (2014) 17011709. 
[3] T. Takekiyo, K. Yamazaki, E. Yamaguchi, H. Abe, Y. Yoshimura, J. Phys. Chem. B, 116 (2012) 1109211097.

[4] P.R. Burney, J. Pfaendtner, J. Phys. Chem. B, 117 (2013) 2662-2670.

[5] D. Constantinescu, C. Herrman, H. Weingartner, Phys. Chem. Chem. Phys., 12 (2010) 1756-1763.

[6] N. Debeljuh, C.J. Barrow, N. Byrne, Phys. Chem. Chem. Phys., 13 (2011) 16534-16536.

[7] O.C. Fiebig, E. Mancini, G. Caputo, T.D. Vaden, J. Phys. Chem. B, 118 (2014) 406-412.

[8] A.M. Figueiredo, J. Sardinha, G.R. Moore, E.J. Cabrita, Phys. Chem. Chem. Phys., 15 (2013) 1963219643.

[9] M. Haberler, C. Schroder, O. Steinhauser, Phys. Chem. Chem. Phys., 13 (2011) 6955-6969.

[10] W.T. Heller, J. Phys. Chem. B, 117 (2013) 2378-2383.

[11] I. Jha, P. Attri, P. Venkatesu, Phys. Chem. Chem. Phys., 16 (2014) 5514-5526.

[12] H.R. Kalhor, M. Kamizi, J. Akbari, A. Heydari, Biomacromolecules, 10 (2009) 2468-2475.

[13] Y. Shu, M. Liu, S. Chen, X. Chen, J. Wang, J. Phys. Chem. B, 115 (2011) 12306-12314.

[14] L.I.N. Tomé, M. Jorge, J.R.B. Gomes, J.o.A.P. Coutinho, J. Phys. Chem. B, 116 (2012) 1831-1842.

[15] H. Noritomi, K. Minamisawa, R. Kamiya, S. Kato, J. Biomedical Science and Engineering, 4 (2011) 9499.

[16] P. Attri, P. Venkatesu, Phys. Chem. Chem. Phys., 13 (2011) 6566-6575.

[17] S.N. Riduan, Y. Zhang, Chem. Soc. Rev., 42 (2013) 9055-9070.

[18] G.D. Elliot, ACS Symposium Series, 1030 (2010) 95-105.

[19] R.A. Muzzarelli, Marine Drugs, 9 (2011) 1510-1533.

[20] G. Liu, Biophysical Reviews and Letters, 7 (2012) 121-134.

[21] S. Demirci, N. Sahiner, J. Mol. Liq., 194 (2014) 85-92.

[22] F. van Rantwijk, R.A. Sheldon, Chem. Rev., 107 (2007) 2757-2785.

[23] S.Y. Yang, F. Cicoira, R. Byrne, F. Benito-Lopez, D. Diamond, R.M. Owens, G.G. Malliaras, Chem. Comm., 46 (2010) 7972-7974.

[24] M. Naushad, Z.A. Alothman, A.B. Khan, M. Ali, Int. J. Biol. Macromolecules, 51 (2012) 555-560.

[25] A.M. Klibanov, Nature, 409 (2001) 241-246.

[26] E.M. Nordwald, J.L. Kaar, J. Phys. Chem. B, 117 (2013) 8977-8986.

[27] K. Sankaranarayanan, G. Sathyaraj, B.U. Nair, A. Dhathathreyan, J. Phys. Chem. B, 116 (2012) 4175-4180.

[28] V.W. Jaeger, J. Pfaendtner, ACS Chem Biol, DOI: 10.1021/cb3006837 (2013).

[29] K. Sankaranarayanan, B. Sreedhar, B.U. Nair, A. Dhathathreyan, J. Phys. Chem. B, 117 (2013) 1234-1240.

[30] A. Pinkert, K.N. Marsh, S. Pang, M.P. Staiger, Chem. Rev., 109 (2009) 6712-6728.

[31] J.S. Moulthrop, R.P. Swatloski, G. Moyna, R.D. Rogers, Chem. Comm., 41 (2005) 1557-1559.

[32] Y.-A. Gao, Z.-H. Li, J.-M. Du, B.-X. Han, G.-Z. Li, W.-G. Hou, D. Shen, L.-Q. Zheng, G.-Y. Zhang, Chem. Eur. J., 11 (2006) 5875-5880.

[33] S.M. Murray, T.K. Zimlich, A. Mirjafari, R.A. O’Brien, J.H.J. Davis, K.N. West, J. Chem. Eng. Data, 58 (2013) 1516-1522.

[34] A. Kumar, P. Venkatesu, Chem. Rev., 112 (2012) 4283-4307.

[35] T. Vasantha, P. Attri, P. Venkatesu, R.S.R. Devi, J. Phys. Chem. B, 116 (2012) 11968-11978.

[36] A. Kumar, P. Venkatesu, Int. J. Biol. Macromolecules, 63 (2014) 244-253.

[37] B.K. Paul, A. Ganguly, N. Guchhait, J. Photochem. and Photobio. B: Biol., 133 (2013) 99-107.

[38] Y. Fan, S. Zhang, Q. Wang, J. Li, H. Fan, D. Shan, Appl. Spectroscopy, 67 (2013) 648-655.

[39] A. Kumar, P. Venkatesu, Process Biochemistry, 49 (2014) 2158-2169.

[40] Q. Shao, J. Chem. Phys., 139 (2013) 115102.

[41] A. Safavi, F. Farjami, Anal. Biochem., 402 (2010) 20-25. 
[42] S.-F. Wang, T. Chen, Z.-L. Zhang, X.-C. Shen, Z.-X. Lu, D.-W. Pang, K.-Y. Wong, Langmuir, 21 (2005) 9260-9266.

[43] P. Du, S. Liu, P. Wu, C. Cai, Electrochimica Acta, 52 (2007) 6534-6547.

[44] Z. Yang, J. Biotechnology, 144 (2009) 12-22.

[45] J.R. Cann, Biochem., 6 (1967) 3435-3442.

[46] M.D. Schuh, J. Chem. Ed., 65 (1988) 740-741.

[47] E.M. Nordwald, G.S. Armstrong, J.L. Kaar, ACS Catal., 4 (2014) 4057-4064.

[48] R. Liyanage, N. Devarapalli, D.B. Pyland, L.M. Puckett, N.H. Phan, J.A. Starch, M.R. Okimoto, J.

Gidden, W.E. Stites, J.O. Lay, Int. J. Mass Spec., 330-332 (2012) 63-70.

[49] G. Wang, R.R. Abzalimov, C.E. Bobst, I.A. Kaltashov, Proc. Natl. Acad. Sci., 110 (2013) 20087-20092.

[50] L. Konermann, J. Pan, Y.-H. Liu, Chem. Soc. Rev., 40 (2011) 1224-1234.

[51] X. Ding, Y. Wang, Q. Zeng, J. Chen, Y. Huang, K. Xu, Analytica Chemica Acta, 815 (2014) 22-32.

[52] M. Nagai, Y. Nagai, K. Imai, S. Neya, Chirality, 26 (2014) 438-442.

[53] C.M. Jones, J. Chem. Ed., 74 (1997) 1306-1310.

[54] P.A. Sykes, H.-C. Shiue, J.R. Walker, R.C. Bateman Jr., J. Chem. Ed., 76 (1999) 1283-1284.

[55] D.E. Otzen, Biophys. J., 83 (2002) 2219-2230.

[56] R. Zwanzig, Proc. Natl. Acad. Sci., 94 (1997) 148-150.

[57] I. Tinoco, K. Sauer, J.C. Wang, J.D. Puglisi, G. Harbison, D. Rovnyak, Physical Chemistry: Principles and Applications in Biological Sciences, 5th ed., Pearson, 2014.

[58] A. Zarrine-Afsar, A.R. Davidson, Methods, 34 (2004) 41-50.

[59] M.M. Patel, F. Tzul, G.I. Makhatadze, Biophysical Chemistry, 159 (2011) 58-65.

[60] S. Roy, B. Bagchi, J. Phys. Chem. B, 118 (2014) 5691-5697.

[61] P. Bharmoria, K.S. Rao, T.J. Trivedi, A. Kumar, J. Phys. Chem. B, 118 (2014) 115-124.

[62] A. Huerta-Viga, S. Woutersen, J. Phys. Chem. Letters, 4 (2013) 3397-3401.

[63] S.K. Jha, S. Marqusee, Proc. Natl. Acad. Sci., 111 (2014) 4856-4861.

[64] Y. Jeon, J. Sung, D. Kim, C. Seo, H. Cheong, Y. Ouchi, R. Ozawa, H.-o. Hamaguchi, J. Phys. Chem. B, 112 (2008) 923-928.

[65] S. Cha, M. Ao, W. Sung, B. Moon, B. Ahlstrom, P. Johansson, Y. Ouchi, D. Kim, Phys. Chem. Chem. Phys., 16 (2014) 9591-9601.

[66] P. Yee, J.K. Shah, E.J. Maginn, J. Phys. Chem. B, 117 (2013) 12556-12566. 


\section{Figure Captions}

Figure 1. Thermal unfolding of $0.2 \mathrm{mg} / \mathrm{mL}$ myoglobin in $\mathrm{pH}-7$ phosphate buffer in the presence of different ILs. The y-axis is the fraction of folded myoglobin computed from the initial and final $409 \mathrm{~nm}$ absorbance values. Each data set is an average of 3 experiments, and a representative data set with error bars is shown in Figure S2. A) Thermal unfolding in increasing concentrations of EMIAc; B) Thermal unfolding in increasing concentrations of TMGAc; C) Thermal unfolding in increasing concentrations of PyrrBF 4 ; D) Thermal unfolding in increasing concentrations of $\mathrm{BMIBF}_{4}$.

Figure 2. Unfolding of $0.2 \mathrm{mg} / \mathrm{mL}$ myoglobin in $\mathrm{pH}=7$ phosphate buffer in the presence of different ionic liquids, each shown as a function of increasing urea concentration. The y-axis is the fraction of folded myoglobin computed from the absorbance at $409 \mathrm{~nm}$ based on initial and final absorbance values. Data shown is an average of two experiments.

Figure 3. Urea-induced myoglobin unfolding kinetics plots with $8.0 \mathrm{M}$ urea in the presence of increasing IL concentration. The fraction unfolded values are computed by normalizing the $409 \mathrm{~nm}$ absorbance values to the value at time zero (which assumes the fraction is 1 before unfolding) and correcting for the remaining absorbance value after unfolding (which assumes the fraction is 0 after unfolding). Each data set is an average of 3 experiments, and a representative data set with error bars is shown in Figure S2. A) Unfolding in increasing concentrations of EMIAc; B) Unfolding in increasing concentrations of TMGAc; C) Unfolding in increasing concentrations of $\mathrm{PyrrBF}_{4}$; D) Unfolding in increasing concentrations of $\mathrm{BMIBF}_{4}$.

Figure 4. Room-temperature $\mathrm{k}_{\mathrm{OBS}}$ rate constants obtained by fitting the data in Figure 3. Error bars are computed based on errors in the exponential fits and are shown as \pm 1 standard deviation. (A) Values shown as a function of increasing IL concentration; (B) Values shown as a function of $\mathrm{I}^{1 / 2}$ to illustrate that some ILs have effects due to ionic strength (in which case kOBS vs $\mathrm{I}^{1 / 2}$ is linear) while other ILs have extra effects. 
Figure 5. Arrhenius plots of the $\mathrm{k}_{\mathrm{OBS}}$ values measured for myoglobin unfolding in $8.0 \mathrm{M}$ urea at different temperatures from 25 to $55{ }^{\circ} \mathrm{C}$. Only the plots for myoglobin in no $\mathrm{IL}, \mathrm{NaCl}$, and $\mathrm{BMIBF}_{4}$ are shown; all plots are shown in Figure S2.

Figure 6. MS of myoglobin (+16 charge state) before and after deuterium exchange (HDX). A) $1 \mathrm{mg} / \mathrm{mL}$ myoglobin in pH-7 phosphate buffer; B) $1 \mathrm{mg} / \mathrm{mL}$ myoglobin in $\mathrm{pH}-7$ phosphate buffer and $200 \mathrm{mM}$ $\mathrm{BMIBF}_{4}$.

Figure 7. Average protein mass after initiation of $\mathrm{HDX}$ experiment shown vs time after adding $\mathrm{D}_{2} \mathrm{O}$, for myoglobin in $\mathrm{pH}-7$ phosphate buffer with different ILs present at $300 \mathrm{mM}$. The HDX reaction was quenched with low-pH buffer after specific mixing times immediately prior to LCMS injection. The measurement at $\mathrm{t}=0$ seconds corresponds to the ESI-MS performed immediately prior to $\mathrm{D}_{2} \mathrm{O}$ addition. 


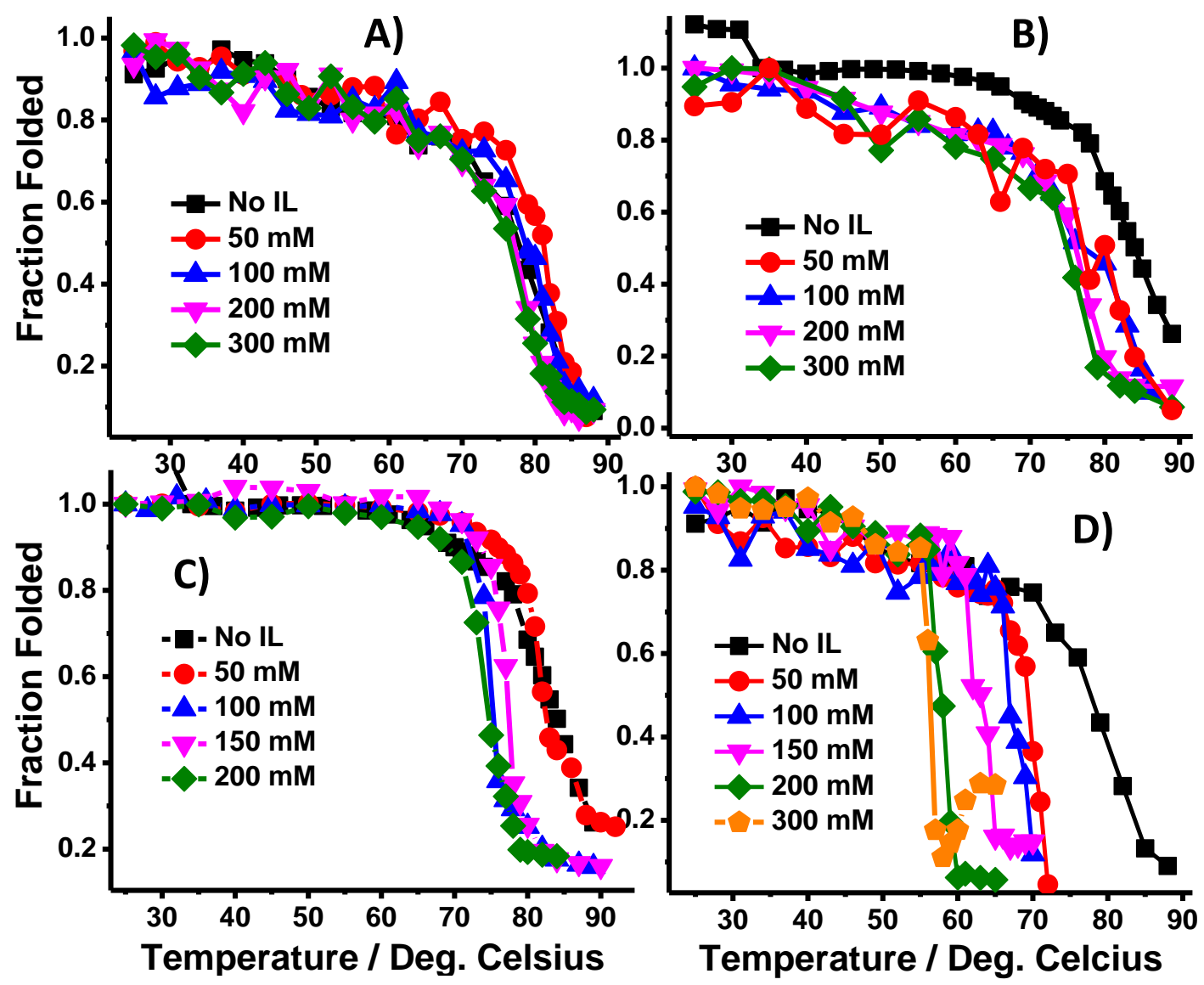

Figure 1 


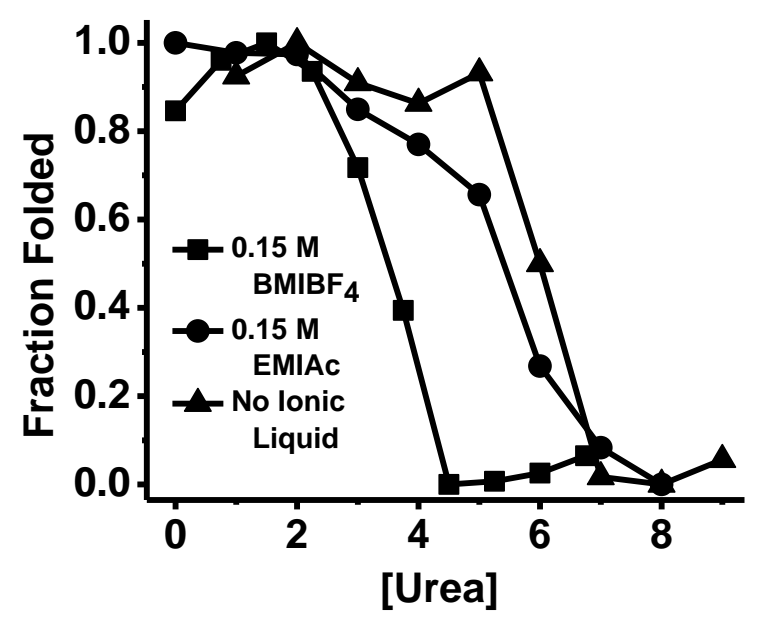

Figure 2 


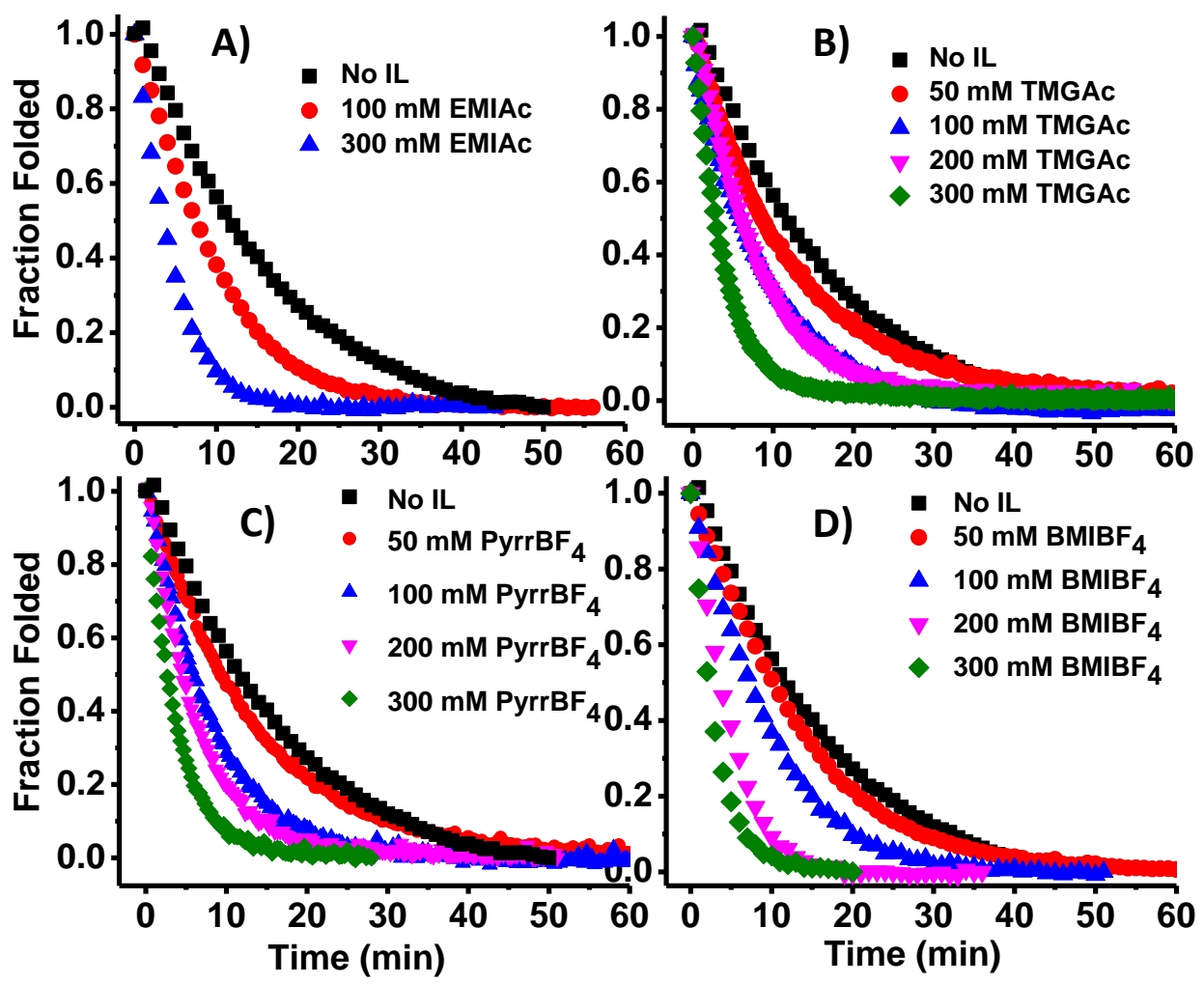

Figure 3 


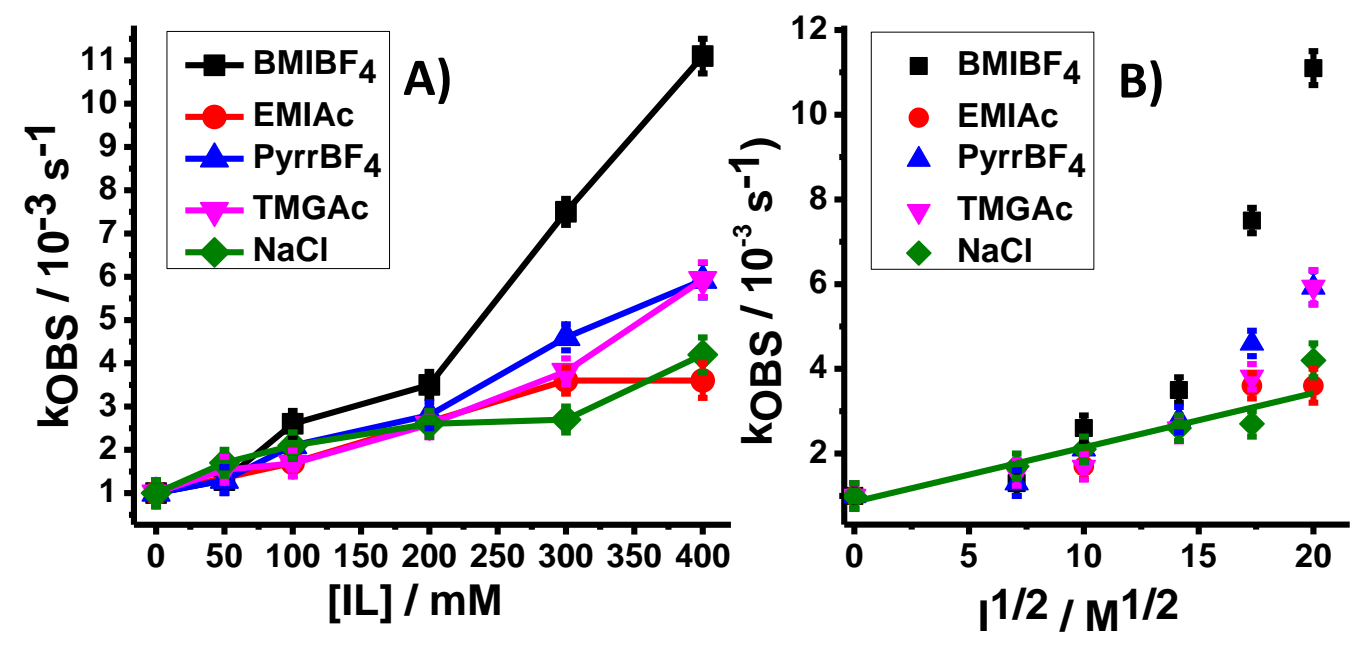

Figure 4 


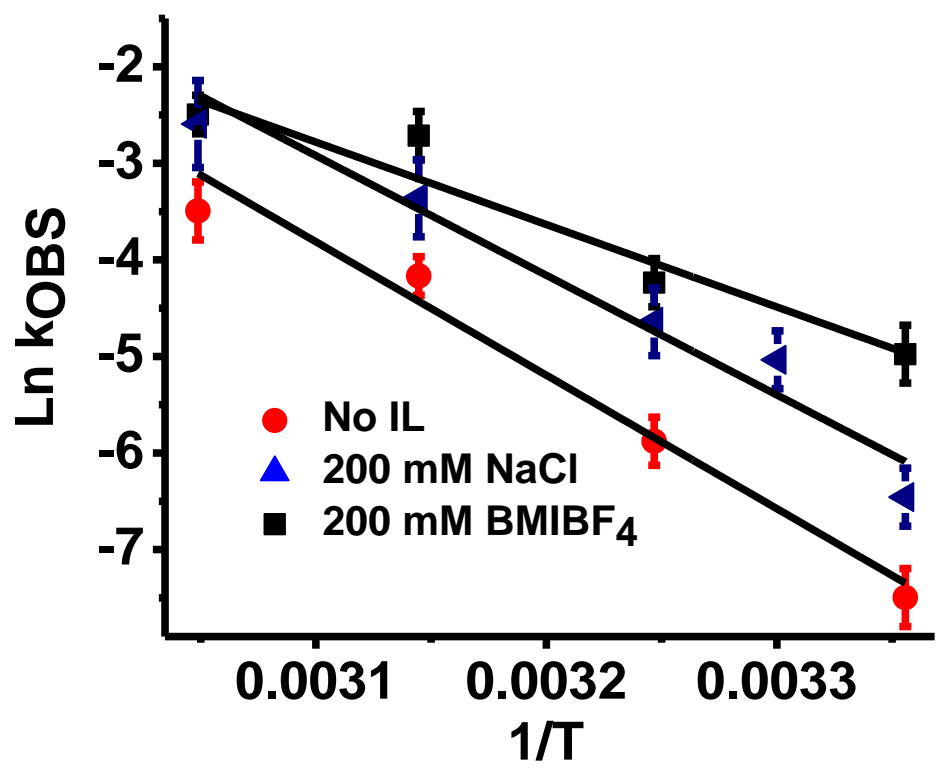

Figure 5 


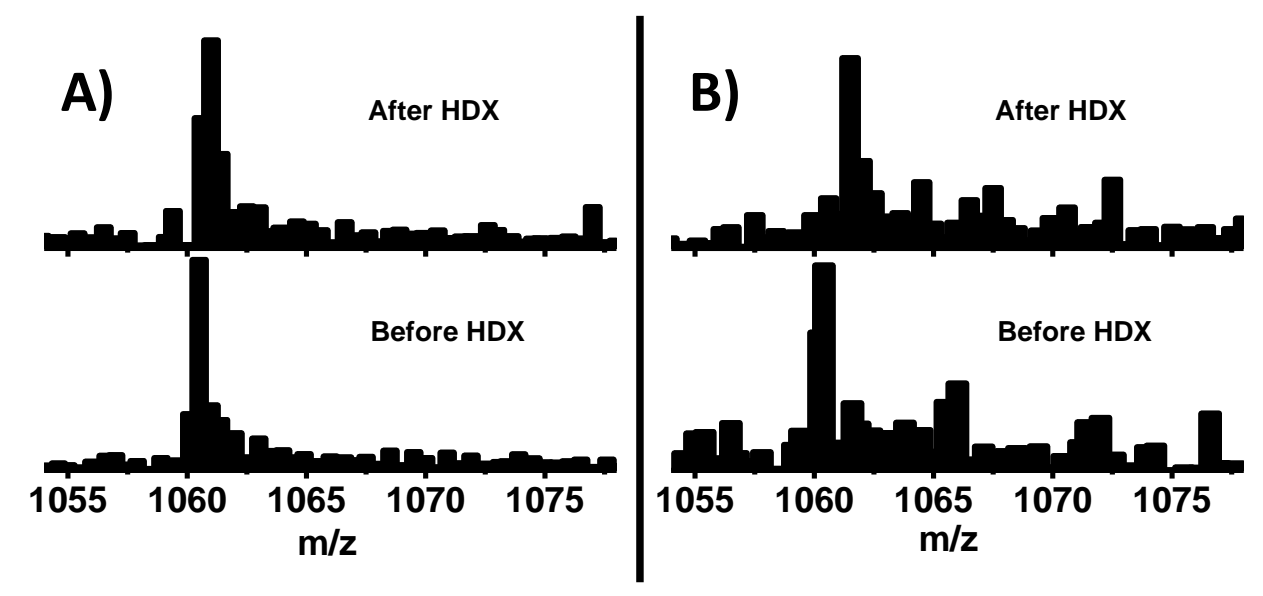

Figure 6 


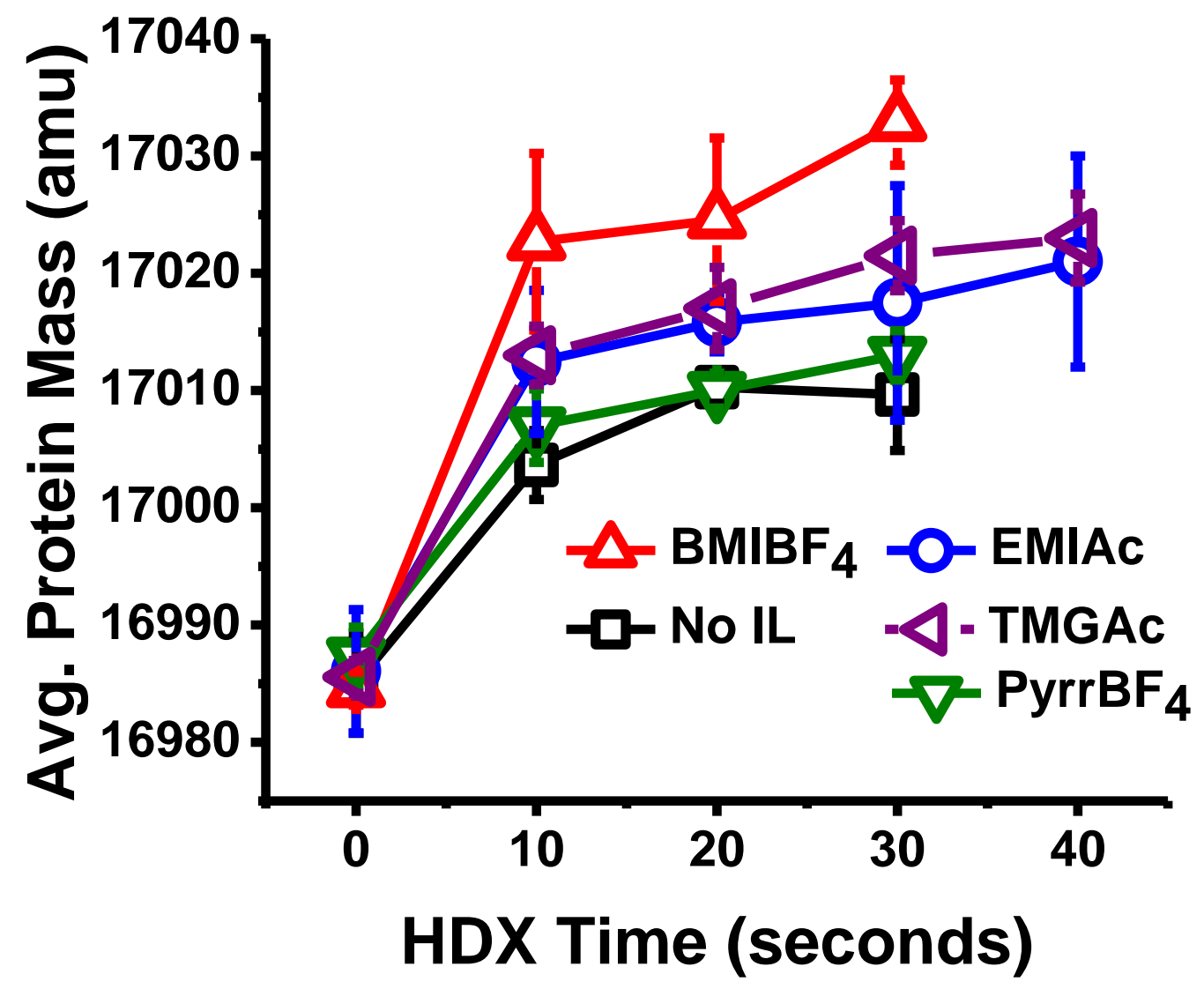

Figure 7 